\title{
Summary of $\alpha_{s}$ Determinations at ZEUS
}

\author{
Claudia Glasman * \\ Universidad Autónoma de Madrid, Spain \\ On behalf of the ZEUS Collaboration
}

\begin{abstract}
The jet cross-section and structure-function measurements done with the ZEUS detector to extract the strong coupling constant and to test its energy-scale dependence are summarised. The values of $\alpha_{s}$ thus obtained and the HERA average are also presented.
\end{abstract}

\section{Introduction}

The strong coupling constant, $\alpha_{s}$, is one of the fundamental parameters of QCD. However, its value is not predicted by the theory and has to be determined experimentally. The success of perturbative QCD (pQCD) lies on precise and consistent determinations of the coupling from many diverse phenomena such as $\tau$ decays, event shapes, $Z$ decays, etc. At ZEUS, many precise determinations of $\alpha_{s}$ have been performed from a variety of measurements based on jet observables and on structure functions.

The procedure to determine $\alpha_{s}$ from jet observables used by ZEUS is based on the $\alpha_{s}$ dependence of the pQCD calculations and takes into account the correlation with the proton parton distribution functions (PDFs). The method consists of performing next-to-leadingorder (NLO) calculations using sets of PDFs for which different values of $\alpha_{s}\left(M_{Z}\right)$ were assumed in the fits. A parameterisation of the $\alpha_{s}\left(M_{Z}\right)$ dependence of the theory for the given observable is obtained. Finally, a value for $\alpha_{s}\left(M_{Z}\right)$ is extracted from the measured cross section using such parameterisation. This procedure handles correctly the complete $\alpha_{s^{-}}$ dependence of the NLO calculations (the explicit dependence in the partonic cross section and the implicit dependence from the PDFs) in the fit, while preserving the correlation between $\alpha_{s}$ and the PDFs.

\section{Determinations of $\alpha_{s}\left(M_{Z}\right)$ at ZEUS}

The exclusive dijet cross section in neutral-current (NC) deep inelastic scattering (DIS) has been measured [2] in the Breit frame in the kinematic region given by $470<Q^{2}<$ $20000 \mathrm{GeV}^{2}$, where $Q^{2}$ is the photon virtuality. Two jets with transverse energies $E_{T, \mathrm{~B}}^{\text {jet } 1}>8$ and $E_{T, \mathrm{~B}}^{\mathrm{jet} 2}>5 \mathrm{GeV}$ and pseudorapidity $-1<\eta_{\mathrm{LAB}}^{\text {jet }}<2$ were selected. Figure 1a shows the ratio of the dijet cross section to the total inclusive DIS cross section as a function of $Q^{2}$. The experimental uncertainties are small, $\sim 6 \%$. The theoretical uncertainties are smaller than for the individual cross sections. The measured ratio is described well by the pQCD prediction. The predictions for different values of $\alpha_{s}$ show the sensitivity of this observable to the coupling. From the measured ratio for $Q^{2}>470 \mathrm{GeV}^{2}$, the value

$$
\alpha_{s}\left(M_{Z}\right)=0.1166 \pm 0.0019 \text { (stat.) }{ }_{-0.0033}^{+0.0024} \text { (exp.) }{ }_{-0.0044}^{+0.0057} \text { (th.) }
$$

was extracted. In this determination, the theoretical uncertainties coming from the higher orders dominate.

\footnotetext{
*Ramón y Cajal Fellow.
} 
Inclusive-jet cross sections in NC DIS have been measured [3] in the Breit frame in the kinematic region of $Q^{2}>125 \mathrm{GeV}^{2}$. Events with at least one jet of $E_{T, \mathrm{~B}}^{\text {jet }}>8 \mathrm{GeV}$ and $-2<\eta_{\mathrm{B}}^{\text {jet }}<1.8$ were selected. There are several advantages of inclusive-jet cross sections with respect to dijet cross sections in a QCD analysis. The inclusive-jet cross sections are infrared insensitive; for dijet cross sections asymmetric $E_{T, \mathrm{~B}}^{\mathrm{jet}}$ cuts are necessary to avoid the infrared-sensitive regions where the NLO programs are not reliable. This difficulty is not present in the calculations of inclusive-jet cross sections so these measurements allow tests of pQCD in a larger phase-space region than in dijet production. Furthermore, the theoretical uncertainties are smaller than in dijet cross sections. Figure $1 \mathrm{~b}$ shows the inclusive-jet cross section as a function of $Q^{2}$ for different jet radii, $R$. The measured cross sections are well described by the NLO predictions. The experimental uncertainties are $\sim 5 \%$. A value of $\alpha_{s}$ has been extracted from the inclusive-jet cross section with $R=1$ for $Q^{2}>500 \mathrm{GeV}^{2}$,

$$
\alpha_{s}\left(M_{Z}\right)=0.1207 \pm 0.0014 \text { (stat.) }{ }_{-0.0033}^{+0.0035} \text { (exp.) }{ }_{-0.0023}^{+0.0022} \text { (th.) }
$$

The experimental uncertainties are dominated by the jet energy scale uncertainty, which amounts to $\pm 2 \%$ and the theoretical uncertainties include the terms beyond NLO $( \pm 1.5 \%)$, the uncertainties coming from the proton PDFs $( \pm 0.7 \%)$ and the hadronisation corrections $( \pm 0.8 \%)$. This determination constitutes the most precise at HERA due to the advantages of using inclusive-jet cross sections at high $Q^{2}$, with a total theoretical uncertainty of only $\pm 1.9 \%$.

The inclusive-jet cross section in photoproduction has been measured [4] as a function of $E_{T}^{\text {jet }}$ (see Fig. 1c). For these processes, transverse energies of up to $95 \mathrm{GeV}$ are accessible. The measured cross section shows a steep fall-off of more than five orders of magnitude within the measured range. The uncorrelated experimental uncertainties are $\sim 5 \%$ at low $E_{T}^{\text {jet }}$ and increase to $\sim 10 \%$ at high $E_{T}^{\text {jet }}$. The theoretical uncertainty due to higher orders is $<10 \%$, and the uncertainties due to the parameterisations of the proton and photon PDFs are $<5 \%$. The hadronisation corrections are $\sim 2.5 \%$ with an uncertainty of $2.5 \%$. The LO calculation underestimates the data by $\sim 50 \%$ for $E_{T}^{\text {jet }}<45 \mathrm{GeV}$, whereas the NLO calculation gives a very good description of the data within the measured range. The determination of $\alpha_{s}$ from inclusive-jet cross sections in photoproduction has an additional uncertainty coming from the photon PDFs, but at the high $E_{T}^{\text {jet }}$ covered by the measurements, the contribution from resolved processes is reduced and so this uncertainty is of the same order as that coming from the proton PDFs. Therefore, this determination of $\alpha_{s}$ is also one of the most precise at HERA,

$$
\alpha_{s}\left(M_{Z}\right)=0.1224 \pm 0.0001 \text { (stat.) }{ }_{-0.0019}^{+0.0022} \text { (exp.) }{ }_{-0.0042}^{+0.0054} \text { (th.). }
$$

The dijet (three-jet) cross sections in NC DIS have been measured [5] as a function of $Q^{2}$ in the Breit frame for events with at least two (three) jets of $E_{T, \mathrm{~B}}^{\mathrm{jet}}>5 \mathrm{GeV}$ and $-1<$ $\eta_{\mathrm{LAB}}^{\mathrm{jet}}<2.5$, in the kinematic range given by $150<Q^{2}<15000 \mathrm{GeV}^{2}$ and $0.2<y<0.6$, where $y$ is the inelasticity. Events with a dijet (trijet) invariant mass $M^{\mathrm{jj}}>25\left(M^{3 \mathrm{j}}>25\right)$ $\mathrm{GeV}$ were selected. Figure 2a shows the ratio of the trijet to the dijet cross section as a function of $Q^{2}$. The data are compared to the predictions of NLO QCD using different values of $\alpha_{s}\left(M_{Z}\right)$. This comparison shows the sensitivity of the observable to the value of $\alpha_{s}$. The measured ratio is described well by the predictions. This ratio is well suited to determine $\alpha_{s}$ at low $Q^{2}$ since the correlated experimental and theoretical uncertainties cancel partially in the ratio. Therefore, this observable provides an accurate test of color dynamics at low $Q^{2}$, 


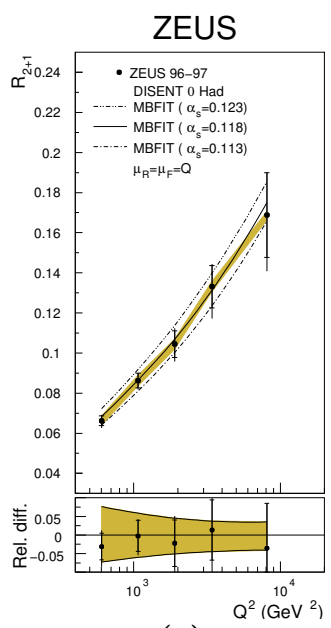

(a)

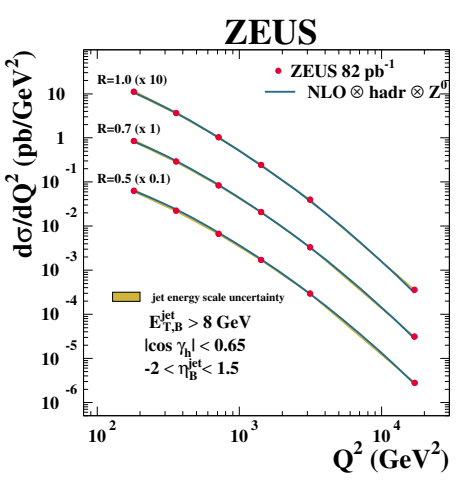

(b)

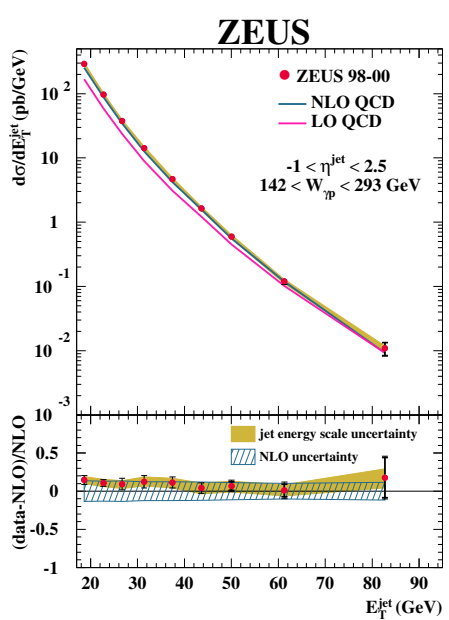

(c)

Figure 1: (a) Normalised dijet cross section as a function of $Q^{2}$ in NC DIS; (b) Inclusive-jet cross section as a function of $Q^{2}$ in NC DIS; (c) Inclusive-jet cross section as a function of $E_{T}^{\mathrm{jet}}$ in photoproduction.

since the theoretical uncertainty of the ratio is of the same order as at higher $Q^{2}$. From the measured ratio of trijet to dijet cross sections in the range $10<Q^{2}<5000 \mathrm{GeV}^{2}$, a value of $\alpha_{s}$ has been extracted,

$$
\alpha_{s}\left(M_{Z}\right)=0.1179 \pm 0.0013 \text { (stat.) }{ }_{-0.0046}^{+0.0028} \text { (exp.) }{ }_{-0.0046}^{+0.0064} \text { (th.), }
$$

with only $\sim 5 \%$ uncertainty coming from higher orders at these low values of $Q^{2}$.

An independent method to extract $\alpha_{s}$ has been developed which relies on the detailed description of the internal structure of the jets by pQCD. The internal structure of jets can be studied by means of the integrated jet shape, which is defined as the average fraction of the jet transverse energy that lies inside a cone in the $\eta-\varphi$ plane of radius $r$ concentric with the jet axis. The integrated jet shape has been measured [6] in NC DIS in the kinematic region given by $Q^{2}>125 \mathrm{GeV}^{2}$ for jets of $E_{T}^{\text {jet }}>17 \mathrm{GeV}$ and $-1<\eta_{\mathrm{LAB}}^{\text {jet }}<2.5$. Figure $2 \mathrm{~b}$ shows the measurements of the mean integrated jet shape as a function of $E_{T}^{\text {jet }}$ for a fixed value of $r=0.5$. The measured integrated jet shape increases as $E_{T}^{\text {jet }}$ increases. The experimental uncertainties and the corrections for detector and hadronisation effects are small for $r=0.5$. The NLO QCD calculations give a very good description of the data and show the sensitivity of this observable to the value of $\alpha_{s}\left(M_{Z}\right)$. The extraction of $\alpha_{s}$ from the internal structure of jets gives a value with one of the smallest experimental uncertainties and negligible theoretical uncertainty coming from the PDFs, but, on the other hand, the theoretical uncertainty from the higher orders increases to about $7 \%$. The value obtained is

$$
\alpha_{s}\left(M_{Z}\right)=0.1176 \pm 0.0009 \text { (stat.) }{ }_{-0.0026}^{+0.0009} \text { (exp.) }{ }_{-0.0072}^{+0.0091} \text { (th.). }
$$

A fit to inclusive DIS data, such as it is shown in Fig. 2c, and jet data has been performed [7] to extract simultaneously the proton PDFs and $\alpha_{s}$. Conventionally, proton PDFs 


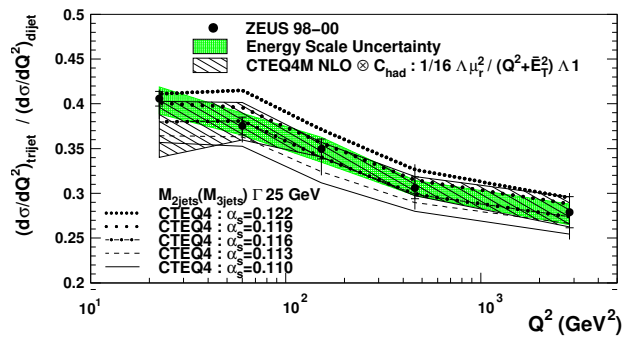

(a)

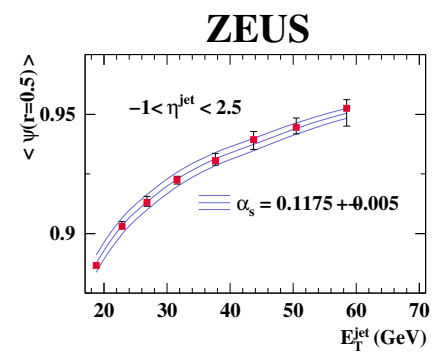

(b)

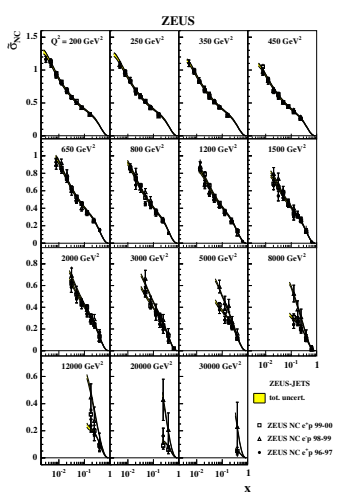

(c)

Figure 2: (a) Ratio of trijet to dijet cross sections as a function of $Q^{2}$ in NC DIS. (b) Mean integrated jet shape as a function of $E_{T}^{\text {jet }}$ in NC DIS; (c) Reduced cross section as a function of $x$ in different regions of $Q^{2}$ in NC DIS.

parameterisations are extracted by fitting inclusive DIS data, which are directly sensitive to the quark content of the proton; the gluon density is extracted via scaling violations and sum rules. On the other hand, jet cross sections are directly sensitive to both the quark and gluon densities and to $\alpha_{s}\left(M_{Z}\right)$, with the processes $\gamma^{(*)} q \rightarrow q g$ not coupled to the gluon density. Therefore, the inclusion of jet cross sections in the fit constraints significantly the gluon density and allows an extraction of $\alpha_{s}\left(M_{Z}\right)$ from structure functions which is not strongly correlated to the gluon density. The value obtained from such a fit is

$$
\alpha_{s}\left(M_{Z}\right)=0.1183 \pm 0.0028 \text { (exp.) } \pm 0.0008 \text { (model) } \pm 0.0050 \text { (h.o.), }
$$

which yields a very precise determination of $\alpha_{s}\left(M_{Z}\right)$ from ZEUS data alone.

Figure 3a shows a summary of the values of $\alpha_{s}\left(M_{Z}\right)$ mentioned above together with other determinations done at ZEUS. All these values are in agreement with each other and with the world average [8]. The experimental uncertainty for the determinations presented here ranges from 1.8 to $4.1 \%$, whereas the theoretical uncertainty is between 1.9 and $7.7 \%$. The value with the lowest theoretical uncertainty is that extracted from the inclusive-jet cross sections in NC DIS.

\section{An average of $\alpha_{s}\left(M_{Z}\right)$ at HERA}

To make a proper average of the determinations of $\alpha_{s}\left(M_{Z}\right)$ from the ZEUS and H1 Collaborations, the correlations among the different determinations has to be taken into account. The experimental contribution to the uncertainty due to that of the energy scale of the jets, which is the dominant source in the jet measurements, is correlated among the determinations from each experiment. On the theoretical side, the uncertainty coming from the proton PDFs is certainly correlated whereas that coming from the hadronisation corrections is only partially correlated. The uncertainty coming from terms beyond NLO is correlated up to a certain, a priori unknown, degree; since these uncertainties are dominant, special care must be taken in the treatment of these uncertainties when making an average of the determinations of $\alpha_{s}\left(M_{Z}\right)$ at HERA. 


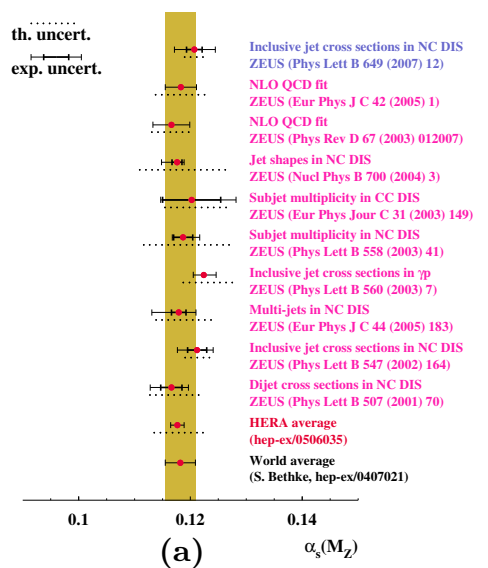

(a)

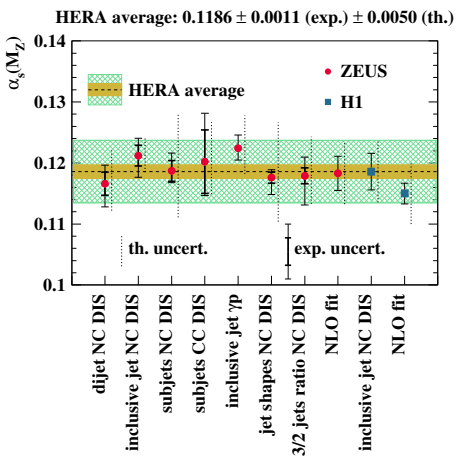

(b)

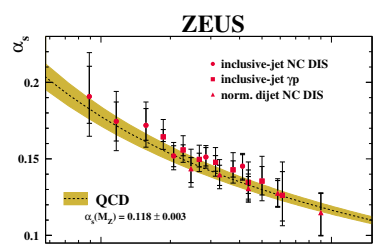

$(\mathbf{c})^{\mu=Q \text { or } \text { E }_{\mathrm{T}}^{\text {it }}(\mathrm{GeV})}$ HERA

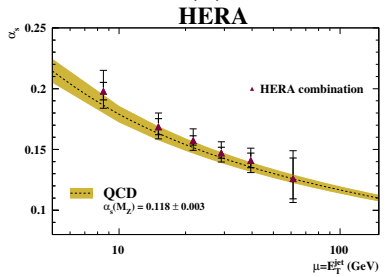

(d)

Figure 3: (a) Summary of $\alpha_{s}\left(M_{Z}\right)$ measurements at ZEUS; (b) HERA $\alpha_{s}\left(M_{Z}\right)$ average; (c) Energy-scale dependence of $\alpha_{s}$ at ZEUS; (d) HERA-combined energy-scale dependence of $\alpha_{s}$.

A conservative approach has been used to make the average [9] in which the known correlations among the determinations of $\alpha_{s}$ coming from the same experiment were taken into account ("correlation method"). The theoretical uncertainties arising from terms beyond NLO were assumed to be (conservatively) fully correlated. Error-weighted averages were obtained separately for the ZEUS and H1 measurements. Finally, a HERA average was obtained by using the error-weighted average method on the ZEUS and H1 averages, assuming the experimental uncertainties to be uncorrelated and taking the overall theoretical uncertainty as the linear average of its contribution in each experiment. The average of the HERA measurements and its uncertainty are [9]:

$$
\overline{\alpha_{s}\left(M_{Z}\right)}=0.1186 \pm 0.0011 \text { (exp.) } \pm 0.0050 \text { (th.), }
$$

with an experimental (theoretical) uncertainty of $\sim 0.9(4) \%$. This average, together with the individual values considered, is shown in Fig. 3b. It is found to be in good agreement with the world average (see Fig. 3a), which does not include any of these determinations.

\section{Energy-scale dependence of $\alpha_{s}$ at ZEUS and HERA combination}

The ZEUS Collaboration has tested the pQCD prediction of the energy-scale dependence of the strong coupling constant by determining $\alpha_{s}$ from the measured differential jet cross sections at different scales $[2,3,4]$. Figure $3 \mathrm{c}$ shows the determinations of the energy-scale dependence of $\alpha_{s}$ as a function of $E_{T}^{\text {jet }}$ or $Q$. The determinations are consistent with the running of $\alpha_{s}$ as predicted by pQCD over a large range in the scale.

The determinations of $\alpha_{s}\left(E_{T}^{\text {jet }}\right)$ from the H1 and ZEUS Collaborations at similar $E_{T}^{\text {jet }}$ have been combined [9] using the correlation method explained above. The combined HERA determinations of the energy-scale dependence of $\alpha_{s}$ are shown in Fig. 3c, in which the running of $\alpha_{s}$ from HERA jet data alone is clearly observed. 


\section{References}

[1] Slides:

http://indico. cern. ch/contributionDisplay $\cdot$ py? contribId=287\&sessionId=17\&conf Id=9499

[2] ZEUS Collaboration, J. Breitweg et al., Phys. Lett. B 507 (2001) 70.

[3] ZEUS Collaboration, S. Chekanov et al., Phys. Lett. B 649 (2007) 12.

[4] ZEUS Collaboration, S. Chekanov et al., Phys. Lett. B 560 (2003) 7.

[5] ZEUS Collaboration, S. Chekanov et al., Eur. Phys. Jour. C 44 (2005) 183.

[6] ZEUS Collaboration, S. Chekanov et al., Nucl. Phys. B 700 (2004) 3.

[7] ZEUS Collaboration, S. Chekanov et al., Eur. Phys. Jour. C 42 (2005) 1.

[8] S. Bethke, J. Phys. G 26 (2000) R27. Updated in preprint hep-ex/0407021.

[9] C. Glasman, Proc. of the 13th International Workshop on Deep Inelastic Scattering, S.R. Dasu and W.H. Smith (eds.), p. 689. Madison, USA (2005). Also in preprint hep-ex/0506035. 